\title{
1 ABCB-mediated auxin transport in outer root tissues regulates lateral root
}

\section{spacing in Arabidopsis} \author{
Jian Chen ${ }^{1,2,{ }^{*}}$, Yangjie Hu ${ }^{3,}{ }^{,}$, Pengchao Hao ${ }^{4}$, Yuqin Zhang ${ }^{3}$, Ohad Roth ${ }^{3}$, Maria F. Njo ${ }^{1,2}$, Lieven Sterck ${ }^{1,2}$,
} Yun $\mathrm{Hu}^{5}$, Yunde Zhao ${ }^{5}$, Markus Geisler ${ }^{4}$, Eilon Shani ${ }^{3}$, Tom Beeckman ${ }^{1,2 \#}$, Steffen Vanneste ${ }^{1,2,6 \#}$

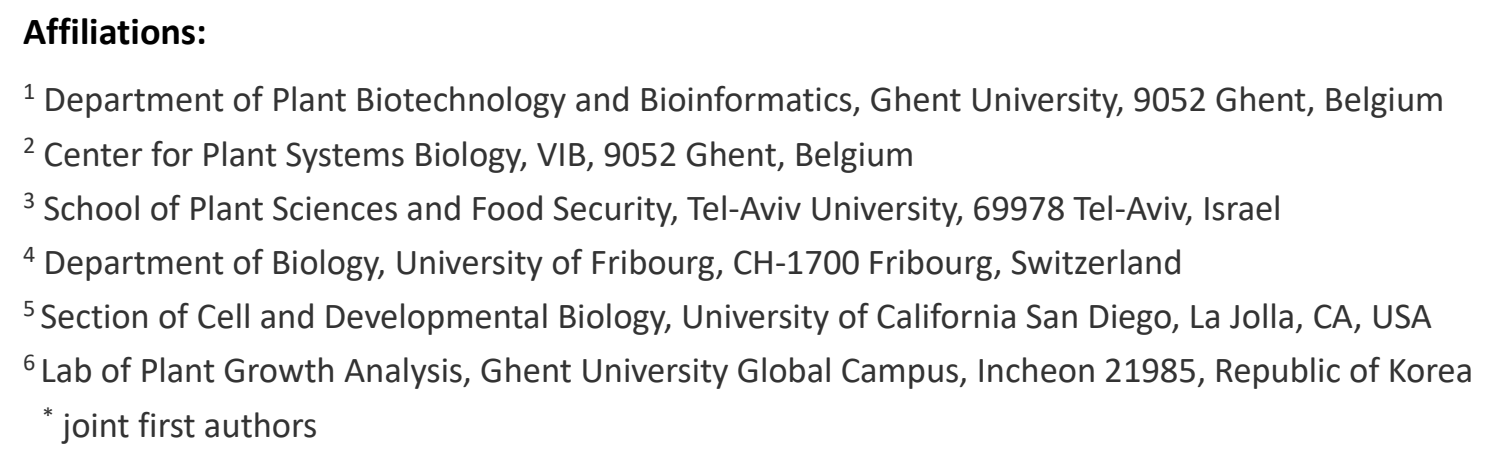

Correspondence: tobee@psb.vib-ugent.be (Tom Beeckman); steffen.vanneste@ugent.be (Steffen Vanneste)

\section{Author contributions}

J.C. generated and analyzed the TAS1c-lines, the phylogenetic tree, the $A B C B$ promotor reporters and genomic YFP-ABCB constructs. Yangjie $\mathrm{H}$. generated and analyzed amiRNA and CRISPR lines, cloned several fluorescent fusion $A B C B$ overexpression lines and performed the auxin inducible cell-type experiments. O.R assisted in amiRNA and CRISPR cloning. Y.Z. identified the amiRNA-2572 line. P.H. performed auxin transport assays. M.F.N made anatomical sections of GUS reporters. L.S. analyzed the sequencing results. Yangjie $\mathrm{H}$. and Yun $\mathrm{H}$. developed the tissue-specific auxin-biosynthesis materials. M.G., E.S., Y.Z., T.B. and S.V. supervised the experiments and data analysis. All authors contributed to the writing of the text. 


\title{
ABCB-mediated auxin transport in outer root tissues regulates lateral root
}

\section{spacing in Arabidopsis}

Jian Chen ${ }^{1,2,{ }^{*}}$, Yangjie Hu ${ }^{3,}{ }^{,}$, Pengchao Hao ${ }^{4}$, Yuqin Zhang ${ }^{3}$, Ohad Roth ${ }^{3}$, Maria F. Njo ${ }^{1,2}$, Lieven Sterck ${ }^{1,2}$, Yun $\mathrm{Hu}^{5}$, Yunde Zhao ${ }^{5}$, Markus Geisler ${ }^{4}$, Eilon Shani ${ }^{3}$, Tom Beeckman ${ }^{1,2 \#}$, Steffen Vanneste ${ }^{1,2,6 \#}$

\begin{abstract}
Root branching is an important strategy to explore efficiently large volumes of soil. To economize this process, lateral roots (LR) are formed along the growing root at discrete positions that are instructed by oscillating auxin signals derived from the lateral root cap (LRC). This assumes that auxin moves from the LRC across multiple layers to accumulate in the pericycle. Here, we identified, using gene silencing and CRISPR based approaches, a group of five genetically linked, closely related ABCBs that control LR spacing by modulating the amplitude of the auxin oscillation. The transporters localize to the plasma membrane and reveal significant auxin export activity. These $A B C B$ s are mainly expressed in the LRC and epidermis where they contribute to auxin transport towards the root oscillation zone. Our findings highlight the importance of auxin transport in the outer tissues of the root meristem to regulate LR spacing.
\end{abstract}

\section{Introduction}

The root system of plants is of vital importance for their growth and survival as it anchors the plant in the soil and is required for the uptake of water and nutrients and symbiotic interactions. The complexity of root systems can be easily expanded by LR branching according to environmentally imposed limitations and stimuli ${ }^{1}$. LR development is a multistep process occurring over a long time, involving coordinated signaling across several tissues ${ }^{2}$. The plant hormone auxin is a key regulator of many organogenetic events in plants ${ }^{3}$. Its local accumulation triggers dramatic, preprogrammed transcriptional changes that are associated with the progression of the developmental program ${ }^{3}$. This is also the case for LR development, where auxin accumulation defines the spacing of prebranch sites along the primary root, and thus root architecture complexity ${ }^{4,5,6,7}$. Therefore, plants have established intricate mechanisms to control auxin distribution within tissues ${ }^{8,9}$, which can be adjusted according to the developmental stage, hormonal and environmental signals ${ }^{1}$. Under normal conditions, the initial 
decision for LR initiation is not made where low-sensitive auxin signaling output reporters in pericycle cells are observed, but rather in a zone more proximal to the meristem ${ }^{6,10}$. In this zone, called the oscillation zone (OZ), oscillatory gene expression was reported to correlate with the activity of the sensitive auxin signaling output reporter $D R 5:: L U C^{5}$. This periodic auxin signaling selects a subset of cells, together denominated as a prebranch site, to gain a higher competence to form a LR reflected in a maintained expression of the auxin output reporter DR5::LUC. ${ }^{5}$. Indole-butyric acid (IBA) to indole5-acetic acid (IAA) conversion in the LRC contributes to the amplitude of this oscillation ${ }^{11,12}$, and cyclic programmed cell death of the LRC contributes to the frequency of this oscillation?

The prevailing model of auxin transport in the meristem can best be summarized as a reverse fountain of auxin flowing rootward through the vascular tissue and being redirected shootward through the outer layers of the meristem ${ }^{13}$. This outer shootward auxin flow is thought to rejoin the central rootward auxin flow (Fig. 1a). The radial inward movement of auxin released from LRC cells undergoing programmed cell death could then contribute to periodic peaks of DR5:LUC that are instructive for $\mathrm{LR}$ positioning ${ }^{7}$. The IAA $/ \mathrm{H}^{+}$symporter AUX1 ${ }^{14}$ represents the major component in the auxin uptake mechanism of the inward radial auxin transport route that controls prebranch site formation ${ }^{6,7}$. However, the corresponding efflux components remain elusive. Currently, the auxin efflux component of the shootward auxin transport in the outer cell layers of the root is believed to be largely explained by the PIN2 auxin transporter ${ }^{15}$, in conjunction with the ABCB-type auxin transporters, $\mathrm{ABCB} 1$ and $\mathrm{ABCB} 19^{16}$. However, neither the pin2 mutant, nor the pin2, abcb1,abcb19 triple mutant showed reduced LR densities or reduced IBA-induced LR formation ${ }^{7}$. This suggests that our current model contains important inaccuracies or shortcomings at least at the level of the molecular identity of the auxin efflux machinery in the root meristem.

Here, we aimed at identifying auxin transporters in the outer tissues of the root that have a role in LR spacing. Therefore, we screened the $A B C B$ transporter family via a tissue-specific gene-silencing approach. We found five closely related $A B C B$ s that are required for $L R$ spacing via modulation of the DR5:LUC oscillation amplitude. These ABCBs localize to the plasma membrane and transport auxin out of the cell. Their predominant expression in LRC and epidermis, and the LR defects in the knockdown/out lines is consistent with these $A B C B$ s acting as effectors of auxin transport in the outer layers of the root meristem that instructs LR spacing. 


\section{Results}

\section{An uncharacterized cluster of five $A B C B$ s controls LR density}

Lateral root initiation and spacing depend on an intricate auxin transport mechanism in the root meristem that can be inhibited via auxin-transport inhibitors such as NPA and BUM ${ }^{7,17,18}$ (Fig. 1a). Because both inhibitors are thought to target, among others, the auxin-transporting $A B C B s^{18,19,20}$, we reasoned that members of the full-sized 22 ABCB protein family ${ }^{21}$ (Fig. 1b) are involved in auxin transport for LR spacing.

To screen the $A B C B$ family for members that are potentially involved in inward radial auxin transport (between the LRC and the pericycle), we used synthetic trans-acting small-interfering RNAs (syn-tasiRNAs) in the AtTAS1c backbone ${ }^{22}$. Based on three syn-tasiRNAs per ABCB subgroup we could theoretically target 21 out of 22 ABCBs: subgroup I: ABCB1,B2,B6,B10,B13,B14,B19,B20 (AtTAS1C$A B C B-I)$, subgroup II: $\mathrm{ABCB} 2, \mathrm{~B} 3, \mathrm{~B} 5, \mathrm{~B} 7, \mathrm{~B} 9, \mathrm{~B} 11, \mathrm{~B} 12, \mathrm{~B} 21$ (AtTAS1C-ABCB-II), and subgroup III: $A B C B 15, B 16, B 17, B 18, B 22$ (AtTAS1C-ABCB-III) (Fig. 1b). For each subgroup, we designed two independent and distinct syn-tasiRNAs to account for variation in silencing efficiency and specificity (indicated as ' $\mathrm{a}$ ' and ' $\mathrm{b}$ ') and expressed them in the LRC, epidermis and cortex via the PIN2 promoter ${ }^{23}$. We determined the LR density of at least two independent, homozygous, single locus lines per construct, relative to WT grown on the same plate, hence analyzing at least 4 independent lines per $A B C B$ subgroup (Fig. 1C). One of the constructs targeting subgroup I $A B C B s$ (AtTAS1c-ABCB-Ib) resulted in a significant reduction of LR density, and both constructs reduced the primary root length (Fig. 1c, Supplementary Fig. 1). None of the AtTAS1C-ABCB-I/ lines showed a significant change in LR density or root length. Interestingly, all tested (six) AtTAS1C-ABCB-III lines showed significant reductions in LR density and root length (Fig. 1c, Supplementary Fig. 1). We could validate the reduction in LR density of 4 independent AtTAS1C-ABCB-III lines, but not the reduction in root length, which was seen in the initial phenotypical screen (Supplementary Fig. 2). This highlights $A B C B 15, B 16, B 17, B 18$ and $B 22$ as potential regulators of LR development. In order to test whether single knockouts would be sufficient to express a LR phenotype, we obtained T-DNA insertion lines in these $A B C B s$. However, none of them showed a defect in root growth and LR formation (Supplementary Fig. 3), indicating a strong functional redundancy as described for other $A B C B$ family members ${ }^{24,25,26,27,28}$. 
Importantly, all members of the subgroup are clustered within $57.6 \mathrm{~kb}$ on chromosome 3 , limiting the generation of multi-knockouts by crossing T-DNA lines. Therefore, to validate the observed AtTAS1C-ABCB-III phenotypes, we followed two independent knock-down/out approaches. On the one hand, we identified, within a collection of artificial microRNA (amiRNA) lines ${ }^{26}$, pro35S::amiR-2572 (named amiR-2572), that targets several of the same $A B C B$ s as the syn-tasi constructs $(A B C B 16, A B C B 17$, $A B C B 22)$ (Supplementary Fig. 4a). On the other hand, we generated an $a b c b^{b 15, b 16, b 17, b 18, b 22}$ mutant via genome editing using 4 sgRNAs, each targeting multiple members of this $A B C B$ subgroup (Supplementary Fig. 4b). We identified one line, named $b 15-b 22^{C R I S P R}$, in which we could not amplify a 3502bp fragment of $A B C B 15$, a 1121 bp fragment of $A B C B 16$, a $1520 \mathrm{bp}$ fragment of $A B C B 17$ and which had a deletion in $A B C B 18$ and a single bp insertion in $A B C B 22$ that causes a premature stop codon, thus likely being a null mutant in these genes (Supplementary Fig. 4c,d). Using Nanostring-based mRNA quantification, we could, however, not reliably detect transcriptional downregulation of any of the targeted $A B C B$ s in our knock-out/down lines, with the exception of $A B C B 15$, being significantly downregulated in $b 15-b 22^{C R I S P R}$ (Supplementary Fig. 4a). Additionally, the non-targeted $A B C B 4$ and $A B C B 19$ were respectively up- and downregulated in $b 15-b 22^{C R I S P R}$ (Supplementary Fig. $4 a$ ) indicating complex interactions among auxin-transporting $A B C B s$, calling for caution in interpreting observed phenotypes.

Both amiR-2572 and $b 15-b 22^{C R I S P R}$ showed a strong reduction in primary root length (Fig. 2b,c,) and LR density (Fig. 2b,d). Moreover, both lines had a smaller rosette size in soil and $b 15-b 22^{C R I S P R}$ had reduced fertility (Supplementary Fig. 4e,f). This suggests that the non-tissue-specific interference with the expression of these $A B C B$ s results in pleiotropic phenotypes. In another genome-editing approach we generated a $54 \mathrm{kbp}$ deletion mutant in which the entire region was deleted, whilst retaining a chimeric fragment of both $A B C B 15$ and $A B C B 22$ (Supplementary Fig 5a,b). In contrast to $b 15-b 22^{C R I S P R}$, this large fragment deletion (Idf-A7) mutant did not display any obvious phenotypes (Supplementary Fig $5 c-f$,), suggesting the induction of a compensation mechanism, eg. similar to the recently described interallelic complementation ${ }^{29}$, or via a compensatory (non)-transcriptional activation of other transport mechanisms. Despite the unresolved issue with the $54 \mathrm{~kb}$ deletion mutant, we argue that 3 independent knock-down constructs and one CRISPR knock-out showing similar LR phenotypes represent compelling evidence for the involvement of the subgroup III ABCBs in LR spacing. 


\section{Subgroup III ABCBs define a new group of plasma membrane-localized auxin exporters}

Because multiple $A B C B s$ have known auxin transport activities ${ }^{26,30,31}$, we postulated that the targeted $A B C B$ subfamily are also bona fide auxin transporters. Therefore, the coding regions of $A B C B 15, B 16$, $B 17, B 18$ and B22 were fused to YFP. Stable transgenic Arabidopsis YFP fusion lines were generated, all showing plasma membrane localization (Fig. 3a). Protoplasts prepared from Agrobacteriumtransfected in $N$. benthamiana leaves showed increased IAA export for all 5 ABCBs at rates comparable to those of the canonical auxin transporting $A B C B 1^{32}$ (Fig. $3 b$ ). In contrast, none of these $A B C B s$ enhanced export of the diffusion control, benzoic acid, (Fig. 3c). Together, the results suggest that the subgroup III $A B C B s$ are plasma membrane localized auxin exporters. In agreement, all 5 ABCBs contained a conserved D/E-P motif in the C-terminal nucleotide binding fold that is diagnostic for auxintransporting $A B C B s^{21}$.

Next, we analyzed the expression pattern of the five subgroup III ABCBs. We found strong expression of $A B C B 15, B 16, B 17$ and $B 22$ in the root meristem (Fig. 4a-d) and for $A B C B 16$ in all stages of LR development (Supplementary Fig. 6a). ABCB18 was almost not expressed in the root meristem, but showed very weak expression in vascular tissues of the hypocotyl and mature root tissues (Fig. 4e; Supplementary Fig. 6c). Besides their root expression, $A B C B 15, B 17$ and $B 22$ were also expressed in cotyledons, leaves and shoot meristem regions (Fig. 4a-d). Interestingly, detailed inspection of the expression pattern using confocal microscopy showed that their root meristematic expression was largely specific to the epidermis and LRC (Fig. 4e,f). This is consistent with a role in the auxin transport

\section{Subgroup III ABCBs control the amplitude of auxin oscillations that instruct LR spacing.}

173 To test the hypothesis that subgroup III ABCBs control LR initiation, we zoomed into the origin of the LR defects of AtTAS1C-ABCB-IIla. For two independent lines, we found that the reduced density of LRs was associated with an overall reduction in the number of LR primordia (LRP). Mainly, the early LRP stages (stages I, II and III) were reduced, without accumulating intermediate stages of LRP development (stages V-VII) (Fig. 5a,b). Similarly, amiR-2572 showed a strong reduction in the early LRP stages, without accumulating intermediate LRP stages (Fig. 2e,f). These data demonstrate that the reduced density of emerged LRs in these lines is due to a defect at the level of initiation. 
by a local maximum of auxin signaling, that can be visualized using DR5:LUC, referred to as prebranch sites $^{5}$. Consistently with the reduced LR initiation, AtTAS1c-IIla showed fewer prebranch sites (Fig. 5ce). The DR5:LUC oscillation period in the AtTAS1C-Illa was similar to that of the wild type (Fig. 5f,g), suggesting that LRC programmed cell death was not affected. In contrast, the DR5:LUC oscillation amplitude in the AtTAS1c-IIla lines was significantly lower than in the WT (Fig. 5f,h), suggesting that not every auxin oscillation develops into a prebranch site and subsequently into a new LR. This suggests that subgroup III $A B C B$ are part of the auxin homeostasis mechanism that determine DR5:LUC oscillation amplitude.

Previously, we inferred from tissue-specific complementation assays and in silico modeling that shootward auxin transport in the LRC is a critical determinant of oscillation amplitude ${ }^{7,11,12}$. The expression patterns of the subgroup III $A B C B$ s and their knock-down phenotypes indicate that they could represent the elusive efflux component in this model. To test this hypothesis, we generated an estradiol-inducible auxin biosynthesis system driven specifically in the quiescence center (QC) ( $p$ WOX5:XVE>>YUC1-2A-TAA1). Simultaneous expression of YUC1 and TAA1 results in IAA synthesis from tryptophan ${ }^{33,34}$. In the control, estradiol treatment induced a strong ectopic DR5:VENUS expression in the LRC, epidermis and the stele in the elongation zone within 7.5h. These effects were further enhanced after 9h estradiol treatment (Fig. 6a). This suggests that the auxin that was produced in the QC, was transported shootward via the LRC and epidermis towards the tissues of the elongation zone, where it activated DR5-VENUS expression. In amiR-2572, the induction of DR5:VENUS in the elongation zone was at both time-points severely reduced compared to the WT (Fig. 6a,b). These data demonstrate that subgroup III $A B C B$ s contribute to shootward auxin transport in the meristem. These data, together with the expression patterns and phenotypes, are consistent with subgroup III-ABCBs being part of the shootward auxin flux in the outer layers of the meristem, that contributes to the DR5:LUC oscillation amplitude and LR spacing.

\section{Discussion}

At its core, spacing of LRs in Arabidopsis can be simplified as the periodic activation of auxin signaling in the pericycle ${ }^{5,6,12}$. This model of LR spacing, assumes a local build-up of auxin that triggers LR initiation when an auxin signaling threshold is surpassed ${ }^{12}$. Surprisingly, the LRC plays a central role in 
to the overall pool of auxin in the meristem via the local conversion of IBA to IAA ${ }^{11}$. On the other hand, dying LRC cells release auxin into the epidermis, resulting in a temporal rise in pericyclic auxin ${ }^{7}$. Periodic cell death in the LRC thus explains the oscillation of auxin activity in the pericycle to instruct LR spacing. In both cases, the LRC contribution to LR spacing assumes auxin transport from the LRC to the pericycle. This auxin transport mechanism involves the AUX1 IAA : $\mathrm{H}^{+}$uptake carrier in the $\mathrm{LRC}^{7}$. Currently, the molecular nature of the auxin efflux machinery involved in LR spacing remains elusive. In an attempt to identify the missing auxin transporter(s), we delved deeper into the potentially massive functional redundancy within the $A B C B$ gene-family, that contains multiple auxin transporters ${ }^{30}$. We identified a group of closely related $A B C B s(A B C B 15, A B C B 16, A B C B 17, A B C B 18$ and $A B C B 22$ ) that are required for $L R$ spacing. We demonstrated that these $A B C B$ s are plasma membranelocalized auxin exporters that contribute to the DR5:LUC oscillation amplitude, via effecting shootward auxin transport in the meristem. Interestingly, these $A B C B$ s are largely co-expressed with $A U X 1$ in the outer tissues of the root meristem, suggesting they act in conjunction with AUX1 in the LRC and epidermis. Although this finding adds a new piece to the puzzle, it remains unclear whether this new cluster of $A B C B s$ auxin transporter also bridges the cortex and endodermis, to feed into the pericyclic auxin pool and to surpass the critical auxin level that triggers LR initiation.

Recently, passive auxin diffusion via plasmodesmata, intercellular pores that linking the cytoplasm of adjacent cells, was shown to markedly improve the accuracy of simulated auxin distribution patterns in the root apical meristem ${ }^{35}$. Therefore, it will be of interest to evaluate the contribution of symplastic connectivity between the radial layers of the root to prebranch site formation and LR spacing.

\section{Materials and methods}

Plant material and growth conditions

Arabidopsis thaliana Colombia (Col-0) ecotype, was used as wild type. abcb15-1 (SALK_034562), $a b c b 16-1$ (SALK_006491), $a b c b 17-1$ (SALK_002801), abcb18-1 (SALK_013774), and abcb22-1 (SALK_202270) mutant seeds were obtained from NASC. Arabidopsis transgenic lines DR5rev:VENUS$N 7^{36}$ and DR5:Luciferase (DR5:LUC $)^{5}$ were crossed and homozygous lines were selected and used as TO for AtTAS1C-ABCBs transformation. Arabidopsis seeds were surface sterilized by chlorine gas, seeds were then sown in Petri dishes $(12 \mathrm{~cm} \mathrm{X} 12 \mathrm{~cm})$ containing sterile half-strength Murashige and Skoog 
medium ( $0.5 \times$ MS salts, $0.8 \%$ sucrose, $0.5 \mathrm{~g} / \mathrm{L} 2$-(N-morpholino) ethanesulfonic acid, $\mathrm{pH} 5.7$, and $0.8 \%$ $w / v$ agar), and grown under continuous light, after 3 days vernalization at $4^{\circ} \mathrm{C}$.

\section{Plasmid construction}

Most constructs were generated by the Gateway system ${ }^{\circledast}$ (Invitrogen, Carlsbad, CA, USA). To construct the YFP fusion, coding sequences amplified from genomic DNA were cloned into pDONR-P2R-P3 (ThermoFisher Scientific) using the primers listed in Supplementary Table 1. The $35 \mathrm{~S}$ driven $\mathrm{N}$-terminal YFP fusion expression clones were constructed by recombining pEN-L4-35S-R1 ${ }^{37}, \mathrm{pEN}-\mathrm{L} 1-\mathrm{Y}-\mathrm{L} 2^{37}$ and the respective CDS clones into $\mathrm{pH} 7 \mathrm{~m} 34 \mathrm{GW}$ using multisite LR Gateway reaction. For the promoter::NLSGFP-GUS reporters, $2 \mathrm{~kb}$ promoter fragments upstream of the coding sequence were amplified from genomic DNA using primers listed in Supplementary Table 2, and subsequently cloned into pENTR $^{\mathrm{TM}}$ TOPO $^{\circledR}$ vector(pENTR ${ }^{\mathrm{TM}} / \mathrm{D}^{-T O P O}{ }^{\circledR}$ Cloning Kits, ThermoFisher Scientific) to generate the corresponding entry clones. The promoter::NLSGFP-GUS was generated by performing an LR recombination reaction between Nuclear GFP fusion (pEN-L1-NF-L2) ${ }^{37}$, GUS reporter (pEN-R2-S*-L3) ${ }^{37}$ and $\mathrm{pH} 7 \mathrm{~m} 34 \mathrm{GW}^{37}$. AtTAS1c-ABCBs constructs were generated using primers TAS-la/b-F/R, TAS-Ila/bF/R and TAS-IIla/b-F/R (Supplementary Table 3) as described ${ }^{22}$. The $p W O X 5: X V E>>Y U C 1-2 A-T A A 1$ construct was generated by cloning the YUC1-2A-TAA1 cassette into Xhol and Spel sites of the pER8 vector ${ }^{38}$. The full-length CDNA of YUC1 was cloned into the BamHI site and the full-length cDNA of TAA1 into the Bglll site of the pM2A vector containing $2 \mathrm{~A}$ peptides ${ }^{39}$. For QC-specific activation of the YUC1-2A-TAA1 cassette, the genomic DNA of WOX5 promoter GCCGTTAACGCTTTCATcgttcagatgtaaagtcctcaactgt) was used.

Generation of $p$ WOX5:XVE>>YUC1-TAA lines pWOX5:XVE>>YUC1-2A-TAA1 ( $p$ WOX5>>YUC1-TAA1) was introduced into DR5:VENUS background by transformation and 10 independent lines were selected. Homozygous lines for both $p$ WOX5 $>>Y C 1$ TAA1 and DR5:VENUS were crossed to amiR-2572 lines to generate F1 seeds. Homozygous plants for pWOX5 >>YUC1-TAA1, DR5:VENUS and amiR-2572 were gained by resistance selection and 
seeds and F3 homozygous for both constructs were obtained.

\section{Agrobacterium and Arabidopsis transformation}

Agrobacterium tumefaciens strain GV3101 was transformed with the relevant binary plasmids via the freeze-thaw procedure ${ }^{40}$. An individual PCR confirmed Agrobacterium colony was used for floral dip ${ }^{41}$. Transformants were selected and the segregation of the $\mathrm{T} 2$ analyzed using appropriate antibiotics.

\section{Phenotyping and LR staging}

To quantify the LR phenotype in wild-type plants and mutants, emerged LR of whole seedlings were counted under a dissecting microscope, 8 days after germination. Root lengths were measured via Fiji (ImageJ $1.52 \mathrm{n}^{42}$ ) using digital images obtained by scanning the Petri dishes.

To analyze the LR primordium stages, root samples were cleared as described previously ${ }^{43}$. All samples were analyzed by differential interference contrast microscopy (Olympus BX51).

\section{Oscillation and prebranch site}

The Luciferase imaging of whole seedlings and oscillation expression analysis was performed as described ${ }^{44}$. A Lumazon FA imaging system (Nippon Roper) carrying a CCD camera from Princeton Instruments Ltd. (Trenton, NJ, USA) or NightSHADE LB985 in vivo plant imaging system (BERTHOLD TECHNOLOGIES) carrying a deep-cooled slow scan CCD camera from Andor Instruments Ltd. (Belfast, UK) were used for luciferase imaging.

To monitor the pre-branch site numbers, we used 8-day-old DR5:LUC seedlings for pre-branch site quantification. The D-luciferin solution (1 mM) was sprayed gently on the seedlings, and kept for $10 \mathrm{~min}$ in the dark and imaged in the Lumazon system with a 15-minute exposure time.

For Long-Term Imaging of Luciferase Signal in the root tip, square plates containing $1 / 2 \mathrm{MS}$ medium were sprayed with $1 \mathrm{mM}$ D-Luciferin solution (0.01\% Tween80) and left to dry in the dark. Then 3-dayold DR5:LUC seedlings were transferred on the plates and imaged immediately with a macro lens every 10 minutes with a 7-minute exposure time for indicated times. The period of the DR5:LUC oscillations 
was determined based on the number of frames that spaced a DR5:LUC maximum in the OZ of each seedling root, multiplied with the time of each cycle.

\section{Kymograph}

Kymographs (http://www.embl.de/eamnet/html/body_kymograph.html) were generated by ImageJ to visualize the spatiotemporal changes of DR5:LUC signal in the root tips during primary root growth. For this purpose, a time-lapse movie (TIFF series) was loaded into ImageJ, and a "Z-projection" was performed to have an overview of the luciferase signal changes following primary root growth over time. Subsequently, a segmented line was drawn on the newly formed primary root and marked by the "ROI manage" function. This line was restored in the original TIFF series to generate "MultipleKymograph". In our experiments, 3- day-old seedlings were used.

\section{Confocal microscopy}

For reporter lines and translational fusion, seedlings were imaged on a Zeiss 710 confocal microscope. For the propidium iodide (PI)-treated root images, seedlings were stained with $2 \mu \mathrm{g} / \mathrm{mL}$ PI for 3 minutes, washed with water, and used for confocal imaging. For root imaging, GFP was excited at $488 \mathrm{~nm}$ and acquired at 500 to $530 \mathrm{~nm}$. YFP was excited at 514 and the emission between 519-564 nm was collected for YFP and between 614-735 $\mathrm{nm}$ for PI. For the $p$ WOX5 $>>Y U C 1-T A A$ experiments seeds were sown on MS plates, stratified at $4^{\circ} \mathrm{C}$ for 2 days, and grown vertically in growth chamber for 4 days at $21^{\circ} \mathrm{C}$. 4-day-old seedlings of the pWOX5:YUC1TAA1, DR5:VENUS in Col-0 and amiR-2572 background were treated with $5 \mu \mathrm{M}$ estradiol for the indicated time-points. Seedlings were stained in $10 \mathrm{mg} \mathrm{L}-1$ propidium iodide for $2 \mathrm{~min}$ and rinsed in water for $30 \mathrm{~s}$. Confocal microscopy was performed using a Zeiss LSM780 inverted confocal microscope equipped with a $20 \times / 0.8 \mathrm{M} 27$ objective lens. VENUS and propidium iodide were excited using an argonion laser and a diode laser, respectively. VENUS was excited at $514 \mathrm{~nm}$ and detected at 518-588 nm, propidium iodide was excited at $561 \mathrm{~nm}$ and detected at $588-718 \mathrm{~nm}$. 
The GUS assay was performed as previously described ${ }^{45}$. For Arabidopsis cross-section root specimens, GUS stained seedlings were subjected to fixation, dehydration and embedding as previously described ${ }^{46}$. GUS-stained tissues were imaged using a Leica Bino and Olympus BX51 microscope for different tissues.

\section{Construction of phylogenetic tree}

The evolutionary history was inferred using the Neighbor-Joining method ${ }^{47}$. The percentage of replicate trees in which the associated taxa clustered together in the bootstrap test with 1000 replicates ${ }^{48}$. The tree is drawn to scale, with branch lengths in the same units as those of the evolutionary distances used to infer the phylogenetic tree. The evolutionary distances were computed using the Maximum Composite Likelihood method and are in the units of the number of base substitutions per site. The analysis involved 21 nucleotide sequences. All positions containing gaps and missing data were eliminated. There were a total of 3455 positions in the final dataset. Evolutionary analyses were conducted in MEGA7 ${ }^{49}$ and optimized via Interaction Tree Of Life (https://itol.embl.de/).

\section{Genotyping}

T-DNA lines for the $A B C B$ single mutants were ordered from The Arabidopsis Information Resource (https://www.arabidopsis.org/), and genotyping primers for T-DNA insertion were designed using the T-DNA Primer Design Tool powered by Genome Express Browser Server (GEBD) (http://signal.salk.edu/tdnaprimers.2.html). Homozygous mutants were selected by PCR performed with primers listed in Supplementary Table 4.

\section{RNA extraction and Nanostring}

Total RNA was isolated from the indicated plant materials using the PureLink RNA Mini Kit (Invitrogen). Nanostring transcript quantification was done as described previously ${ }^{26}$.

\section{Auxin transport measurements}

Simultaneous ${ }^{3} \mathrm{H}$-IAA and ${ }^{14} \mathrm{C}$-benzoic acid (BA) export from tobacco ( $N$. benthamiana) mesophyll protoplasts was analyzed as described ${ }^{19}$. Tobacco mesophyll protoplasts were prepared 4 days after agrobacterium-mediated transfection with proS35S:ABCB1-YFP, pro35S:YFP-ABCB15, pro35S:YFP$A B C B 16$, pro35S:YFP-ABCB17, pro35S:YFP-ABCB18, pro35S:YFP-ABCB22. Relative export from 
protoplasts is calculated from exported radioactivity into the supernatant as follows: (radioactivity in the protoplasts at time $t=10 \mathrm{~min}$.$) - (radioactivity in the supernatant at time t=0)) *(100 \%) /$ (radioactivity in the supernatant at $t=0$ min.); presented are mean values from $>4$ independent transfections.

\section{CRISPR/Cas9 mutagenesis and selection of mutant alleles}

Four single-guide (sg) RNAs were designed using the CRISPR-P tool (http://cbi.hzau.edu.cn/cgibin/CRISPR) ${ }^{50}$ to align the $A B C B s$ coding sequence. The sgRNAs are designed to target multiple $A B C B s$ at once: sgRNA-19 targets ABCB16, 18, 22, 17 (20\% cleavage), and 15 (0.3\% cleavage); sgRNA-20 targets ABCB18, 22, 16, (92\% cleavage), 17 (10\% cleavage) and 15 (0.1\% cleavage); sgRNA3 targets ABCB16, 18,17 ( $0.4 \%$ cleavage), and 15 ( $0.1 \%$ cleavage) and sgRNA4 targets ABCB16, 17,18 ( $49 \%$ cleavage), and 15 ( $0.1 \%$ cleavage). Vectors were assembled using the Golden Gate cloning system ${ }^{51}$. The sgRNA-19, sgRNA-20, sgRNA-3 and sgRNA-4 were cloned downstream of the Arabidopsis U6 promoter (pATU6) in the Level 1 acceptors plCH47761, plCH47772, plCH47781 and plCH47791, respectively, as previously described ${ }^{52}$. The Level 1 constructs were assembled in the binary Level 2 vector pAGM4723. sgRNA sequences are listed in Supplementary Table 5. Genotyping was carried out using primers listed in Supplementary Table 6.

For the large fragment deletion mutant (Idf), 3 sgRNAs were designed targeting ABCB15, and another 3 sgRNAs were designed targeting ABCB22. The sgRNAs are listed in Supplementary Table 7. Six sgRNAs were assembled into pFASTRK as described ${ }^{53}$. Pooled T1 plants were screened for a $0.5-1 \mathrm{~kb}$ amplicon, using primers spanning the $54 \mathrm{~kb}$ genomic fragment, as an indicator of the deletion. In the $\mathrm{T} 2$ generation, individuals lacking the Cas9 transgene were screened for the amplicon, which was sent for Sanger sequencing. Genotyping was done using primers listed in Supplementary Table 8. In order to confirm the correct excision and deletion of the targeted region from the genome, we used NGS. Sequencing was perfomed on an illumina HiSeq 4000 machine, which yielded 47,827,766 reads (150nt PE), being 57.8x coverage. The reads were then aligned to the reference ATH Col-0 genome using BBMap (https://igi.doe.gov/data-and-tools/bbtools/) using default settings. The obtained BAM files containing the aligned reads was subsequently processed with bedtools genomecov ${ }^{54}$ (parameter settings: -bga -split ). This resulted in a coverage plot reflecting the sequencing depth over the ATH 
bioRxiv preprint doi: https://doi org/10.1101/2020.07.22.206300; this version posted July 22, 2020. The copyright holder for this preprin (which was not certified by peer review) is the author/funder, who has granted bioRxiv a license to display the preprint in perpetuity. It is made available under aCC-BY-NC-ND 4.0 International license.

present (indicated by having a coverage of zero ), in our re-sequenced line. Moreover, it also showed no off-target modifications, nor that the excised region would have been reinserted elsewhere in the genome.

Data supporting the NGS analysis part of this study has been deposited at the ENA under BioProject number: PRJEB38980 .

\section{Acknowledgements}

We thank Jose Alonso and Thomas Jacobs for providing early access to unpublished materials at the beginning of this project. This work was supported by grants from the Swiss National Funds (31003A165877/1) to M.G., the China Scholarship Council to J.C., the European Research Council Starting Grant (757683- RobustHormoneTrans) to E.S., the PBC postdoc fellowship to Y.H and Y.Z.

\section{REFERENCES}

1. Motte H, Vanneste S, Beeckman T. Molecular and Environmental Regulation of Root Development. Annu Rev Plant Bio/ 70, 465-488 (2019).

2. Stoeckle D, Thellmann M, Vermeer JE. Breakout-lateral root emergence in Arabidopsis thaliana. Curr Opin Plant Bio/ 41, 67-72 (2018).

3. Vanneste S, Friml J. Auxin: a trigger for change in plant development. Ce// 136, 1005-1016 (2009).

4. Dubrovsky JG, et al. Auxin acts as a local morphogenetic trigger to specify lateral root founder cells. Proc Natl Acad Sci U S A 105, 8790-8794 (2008).

5. Moreno-Risueno MA, Van Norman JM, Moreno A, Zhang J, Ahnert SE, Benfey PN. Oscillating gene expression determines competence for periodic Arabidopsis root branching. Science 329, 1306 1311 (2010).

6. De Smet I, et al. Auxin-dependent regulation of lateral root positioning in the basal meristem of 
bioRxiv preprint doi: https://doi org/10.1101/2020.07.22.206300; this version posted July 22, 2020. The copyright holder for this preprin (which was not certified by peer review) is the author/funder, who has granted bioRxiv a license to display the preprint in perpetuity. It is made available under aCC-BY-NC-ND 4.0 International license.

Arabidopsis. Development 134, 681-690 (2007).

7. Xuan W, et al. Cyclic programmed cell death stimulates hormone signaling and root development in Arabidopsis. Science 351, 384-387 (2016).

8. Adamowski M, Friml J. PIN-dependent auxin transport: action, regulation, and evolution. Plant Cell 27, 20-32 (2015).

9. Rosquete MR, Barbez E, Kleine-Vehn J. Cellular auxin homeostasis: gatekeeping is housekeeping. Mol Plant 5, 772-786 (2012).

10. De Rybel B, et al. A novel aux/IAA28 signaling cascade activates GATA23-dependent specification of lateral root founder cell identity. Curr Bio/ 20, 1697-1706 (2010).

11. De Rybel B, et al. A role for the root cap in root branching revealed by the non-auxin probe naxillin. Nat Chem Bio/8, 798-805 (2012).

12. Xuan W, et al. Root Cap-Derived Auxin Pre-patterns the Longitudinal Axis of the Arabidopsis Root. Curr Bio/ 25, 1381-1388 (2015).

13. Grieneisen VA, Xu J, Maree AF, Hogeweg P, Scheres B. Auxin transport is sufficient to generate a maximum and gradient guiding root growth. Nature 449, 1008-1013 (2007).

14. Swarup R, Bhosale R. Developmental Roles of AUX1/LAX Auxin Influx Carriers in Plants. Front Plant Sci 10, 1306 (2019).

15. Wisniewska J, et al. Polar PIN localization directs auxin flow in plants. Science 312, 883 (2006).

16. Bailly $\mathrm{A}$, et al. Modulation of P-glycoproteins by auxin transport inhibitors is mediated by interaction with immunophilins. J Biol Chem 283, 21817-21826 (2008).

17. Casimiro I, et al. Auxin transport promotes Arabidopsis lateral root initiation. Plant Cel/ 13, 843852 (2001).

18. Kim JY, et al. Identification of an $\mathrm{ABCB} / \mathrm{P}$-glycoprotein-specific inhibitor of auxin transport by chemical genomics. J Biol Chem 285, 23309-23317 (2010).

19. Henrichs $\mathrm{S}$, et al. Regulation of ABCB1/PGP1-catalysed auxin transport by linker phosphorylation. EMBO J31, 2965-2980 (2012).

20. Teale W, Palme K. Naphthylphthalamic acid and the mechanism of polar auxin transport. J Exp Bot 69, 303-312 (2018).

21. Hao P, et al. A conserved D/E-P motif in the nucleotide binding domain of plant ABCB/PGP-type 
bioRxiv preprint doi: https://doi org/10.1101/2020.07.22.206300; this version posted July 22, 2020. The copyright holder for this preprin (which was not certified by peer review) is the author/funder, who has granted bioRxiv a license to display the preprint in perpetuity. It is made available under aCC-BY-NC-ND 4.0 International license.

ABC transporters defines their auxin transport capacity. (2020).

22. Carbonell A, Takeda A, Fahlgren N, Johnson SC, Cuperus JT, Carrington JC. New generation of artificial MicroRNA and synthetic trans-acting small interfering RNA vectors for efficient gene silencing in Arabidopsis. Plant Physio/ 165, 15-29 (2014).

23. Marques-Bueno MDM, et al. A versatile Multisite Gateway-compatible promoter and transgenic line collection for cell type-specific functional genomics in Arabidopsis. Plant J85, 320-333 (2016).

24. Noh B, Murphy AS, Spalding EP. Multidrug resistance-like genes of Arabidopsis required for auxin transport and auxin-mediated development. Plant Ce//13, 2441-2454 (2001).

25. Wu G, Otegui MS, Spalding EP. The ER-localized TWD1 immunophilin is necessary for localization of multidrug resistance-like proteins required for polar auxin transport in Arabidopsis roots. Plant Cel/ 22, 3295-3304 (2010).

26. Zhang $Y$, et al. A transportome-scale amiRNA-based screen identifies redundant roles of Arabidopsis ABCB6 and ABCB20 in auxin transport. Nat Commun 9, 4204 (2018).

27. Geisler M, et al. TWISTED DWARF1, a unique plasma membrane-anchored immunophilin-like protein, interacts with Arabidopsis multidrug resistance-like transporters AtPGP1 and AtPGP19. Mol Biol Cel/ 14, 4238-4249 (2003).

28. Blakeslee J], et al. Interactions among PIN-FORMED and P-glycoprotein auxin transporters in Arabidopsis. Plant Cel/ 19, 131-147 (2007).

29. Brumos J, Bobay BG, Clark CA, Alonso JM, Stepanova AN. Structure-function analysis of interallelic complementation in ROOTY transheterozygotes. Plant Physiol, (2020).

30. Geisler M, Aryal B, di Donato M, Hao P. A Critical View on ABC Transporters and Their Interacting Partners in Auxin Transport. Plant Cell Physio/ 58, 1601-1614 (2017).

31. Kaneda $\mathrm{M}$, et al. $\mathrm{ABC}$ transporters coordinately expressed during lignification of Arabidopsis stems include a set of ABCBs associated with auxin transport. J Exp Bot 62, 2063-2077 (2011).

32. Geisler M, et al. Cellular efflux of auxin catalyzed by the Arabidopsis MDR/PGP transporter AtPGP1. Plant J 44, 179-194 (2005).

33. Mashiguchi K, et al. The main auxin biosynthesis pathway in Arabidopsis. Proc Natl Acad Sci U S A 108, 18512-18517 (2011).

34. Won $\mathrm{C}$, et al. Conversion of tryptophan to indole-3-acetic acid by TRYPTOPHAN AMINOTRANSFERASES OF ARABIDOPSIS and YUCCAs in Arabidopsis. Proc Natl Acad Sci U S A 108, 18518-18523 (2011). 
bioRxiv preprint doi: https://doi org/10.1101/2020.07.22.206300; this version posted July 22, 2020. The copyright holder for this preprin (which was not certified by peer review) is the author/funder, who has granted bioRxiv a license to display the preprint in perpetuity. It is made available under aCC-BY-NC-ND 4.0 International license.

501

502

503

504

505

506

507

508

509

510

511

512

513

514

515

516

517

518

519

520

521

522

523

524

525

526

527

528

529

530

531

532

533

534

535

536

537

538

539

540

541

542

543

544
35. Mellor NL, Voss U, Janes G, Bennett MJ, Wells DM, Band LR. Auxin fluxes through plasmodesmata modify root-tip auxin distribution. Development 147, (2020).

36. Heisler MG, et al. Patterns of auxin transport and gene expression during primordium development revealed by live imaging of the Arabidopsis inflorescence meristem. Curr Bio/15, 1899-1911 (2005).

37. Karimi M, Bleys A, Vanderhaeghen R, Hilson P. Building blocks for plant gene assembly. Plant Physiol 145, 1183-1191 (2007).

38. Zuo J, Niu QW, Chua NH. Technical advance: An estrogen receptor-based transactivator XVE mediates highly inducible gene expression in transgenic plants. Plant J 24, 265-273 (2000).

39. $\mathrm{Kim} \mathrm{JH}$, et al. High cleavage efficiency of a 2A peptide derived from porcine teschovirus-1 in human cell lines, zebrafish and mice. PLoS One 6, e18556 (2011).

40. Chen H, Nelson RS, Sherwood JL. Enhanced recovery of transformants of Agrobacterium tumefaciens after freeze-thaw transformation and drug selection. Biotechniques 16, 664-668, 670 (1994).

41. Clough SJ, Bent AF. Floral dip: a simplified method for Agrobacterium-mediated transformation of Arabidopsis thaliana. Plant J16, 735-743 (1998).

42. Schindelin J, Rueden CT, Hiner MC, Eliceiri KW. The ImageJ ecosystem: An open platform for biomedical image analysis. Mol Reprod Dev 82, 518-529 (2015).

43. Malamy JE, Benfey PN. Organization and cell differentiation in lateral roots of Arabidopsis thaliana. Development 124, 33-44 (1997).

44. Xuan W, Opdenacker D, Vanneste S, Beeckman T. Long-Term In Vivo Imaging of Luciferase-Based Reporter Gene Expression in Arabidopsis Roots. Methods Mol Bio/1761, 177-190 (2018).

45. Beeckman T, Viane R. Embedding thin plant specimens for oriented sectioning. Biotech Histochem 75, 23-26 (2000).

46. De Smet I, Chaerle P, Vanneste S, De Rycke R, Inze D, Beeckman T. An easy and versatile embedding method for transverse sections. J Microsc 213, 76-80 (2004).

47. Saitou N, Nei M. The neighbor-joining method: a new method for reconstructing phylogenetic trees. Mol Biol Evol 4, 406-425 (1987).

48. Felsenstein J. Confidence Limits on Phylogenies: An Approach Using the Bootstrap. Evolution 39, 783-791 (1985). 
49. Kumar S, Stecher G, Tamura K. MEGA7: Molecular Evolutionary Genetics Analysis Version 7.0 for

50. Lei Y, Lu L, Liu HY, Li S, Xing F, Chen LL. CRISPR-P: a web tool for synthetic single-guide RNA design of CRISPR-system in plants. Mol Plant 7, 1494-1496 (2014).

51. Engler C, et al. A golden gate modular cloning toolbox for plants. ACS Synth Bio/3, 839-843 (2014).

52. Soyk S, et al. Bypassing Negative Epistasis on Yield in Tomato Imposed by a Domestication Gene. Cel/ 169, 1142-1155 e1112 (2017).

53. Decaestecker W, et al. CRISPR-TSKO: A Technique for Efficient Mutagenesis in Specific Cell Types, Tissues, or Organs in Arabidopsis. Plant Cel/ 31, 2868-2887 (2019).

54. Quinlan AR. BEDTools: The Swiss-Army Tool for Genome Feature Analysis. Curr Protoc 


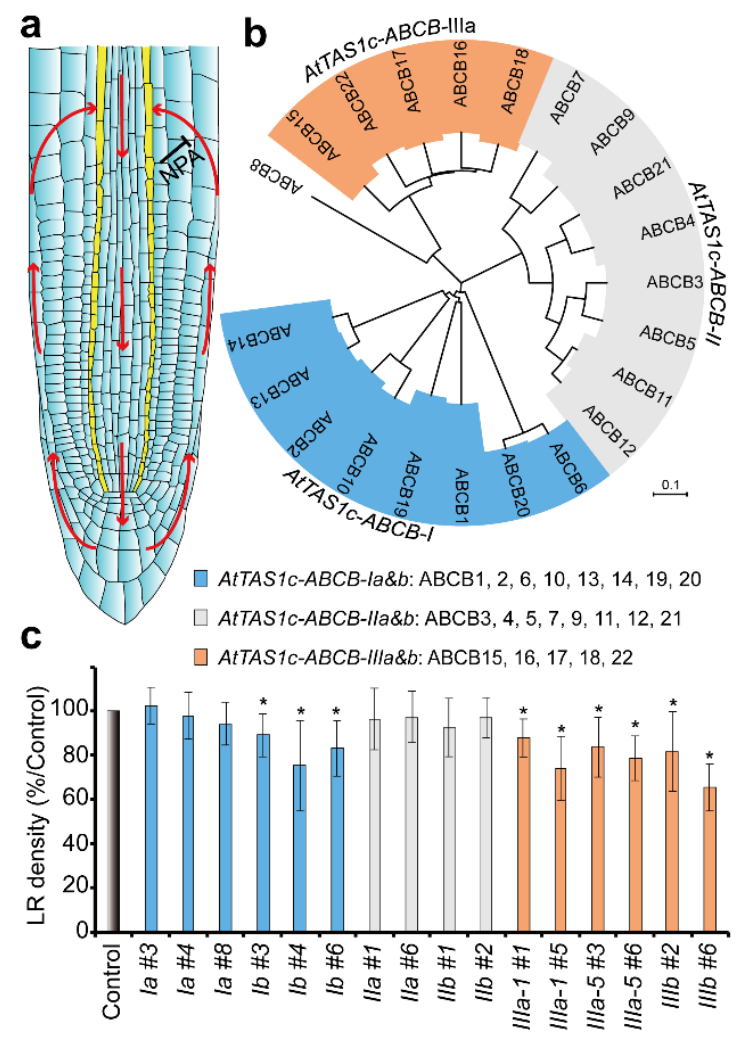

Fig. 1: Phenotypic screen of the $A B C B$ gene family for regulators of LR formation using PIN2 driven syn-tasi-RNA-based gene-silencing. a Reverse fountain model of auxin transport in the Arabidopsis root apical meristem. Red arrows show the major auxin transport directions. The pericycle is indicated in yellow. $\mathbf{b}$ Phylogenetic tree of $A B C B$ gene family. Subgroup genes targeted by different synthetic AtTAS1c constructs are colored blue (AtTAS1C-ABCB-I), grey (AtTAS1C-ABCB-II) and orange (AtTAS1C-ABCB-III). Syn-tasi-RNA constructs are driven by the PIN2 promoter. c Quantification of the emerged lateral root density of 10-day-old AtTAS1c lines relative to WTs grown on the same plate in the initial phenotypic screen, shown are averages $( \pm S D)$ of $>15$ plants. WT controls are set to $100 \%$, * indicates $\mathrm{P}<0.05$ by two-tailed Student's t-test relative to WT. Colors correspond to different subgroups as indicated in $\mathbf{b}$. 


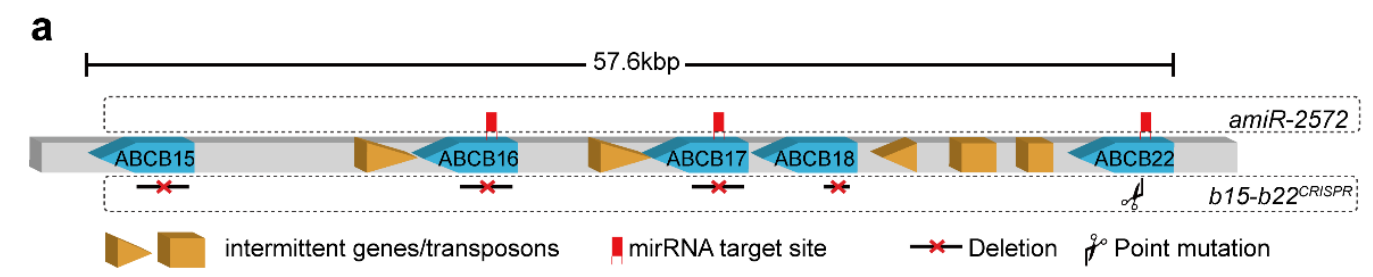

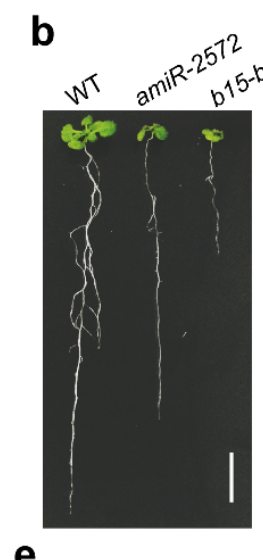

e

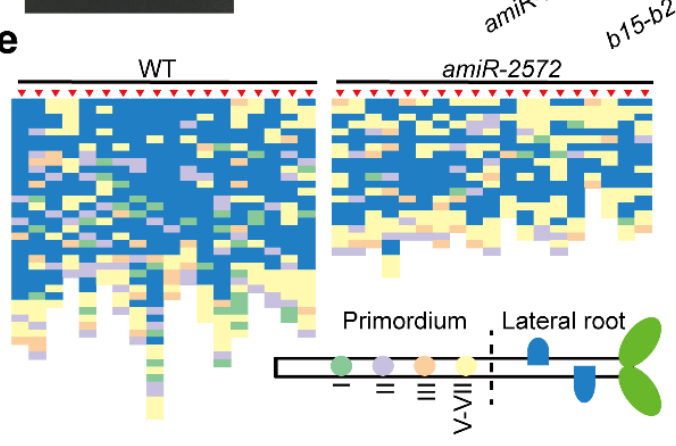

C

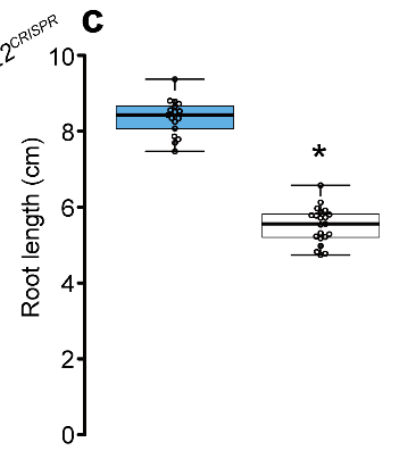$$
\text { WT }
$$

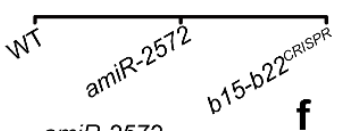

$f$
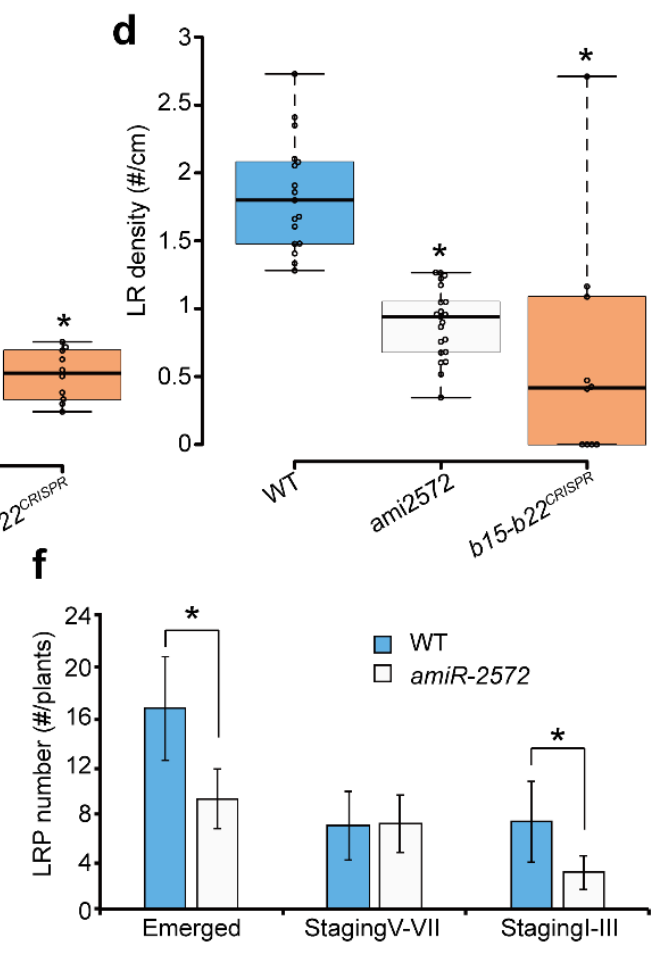

Fig. 2: amiRNA and CRISPR confirm the importance of subgroup III ABCBs in LR development. a Organization of subgroup III $A B C B$ s on the chromosome. All $A B C B$ coding-regions are highlighted in blue, intermittent genes and transposons are highlighted in orange. amiR-2572 target sites in the respective $A B C B$ s are indicated with red marker in the square upper. The deletion within $A B C B 15,16$, 17,18 and the point mutation site of $A B C B 22$ in $b 15-b 22^{C R I S P R}$ are indicated in the square below. $\mathbf{b}$ Macroscopic seedling phenotype of 15-day-old amiR-2572 and b15-b22CRISPR compared to WT. Scale bar is $1 \mathrm{~cm}$. c, d Boxplots showing the quantification of emerged lateral root number (c) and root length (d) in 15-day-old WT, amiR-2572 lines and b15-b22CRISPR mutant seedlings. Center lines show the medians; box limits indicate the 25 th and 75 th percentiles as determined by $R$ software; whiskers extend 1.5 times the interquartile range from the 25 th and 75 th percentiles, outliers are represented by dots; data points are plotted as open circles. $\mathrm{n}=17(\mathrm{WT}), 21$ (amiR-2572), 10 (b15-b22CRISPR), ${ }^{*}$ indicates $\mathrm{P}$ $<0.05$ (two-tailed Student's t-test). e Schematic representation of the distribution of emerged LRs and LRP along the primary root in 8-day-old WT and amiR-2572 seedlings, ( $\mathrm{n}=18$ for WT and 19 for amiR2572). The color code corresponds to different LRP stages as indicated on the schematic root below. $f$ Quantification of different LRP stages and emerged LR per root in $(\mathbf{a})$, shown are averages $( \pm S D)$, * indicates $P<0.05$ (two-tailed Student's t-test). 

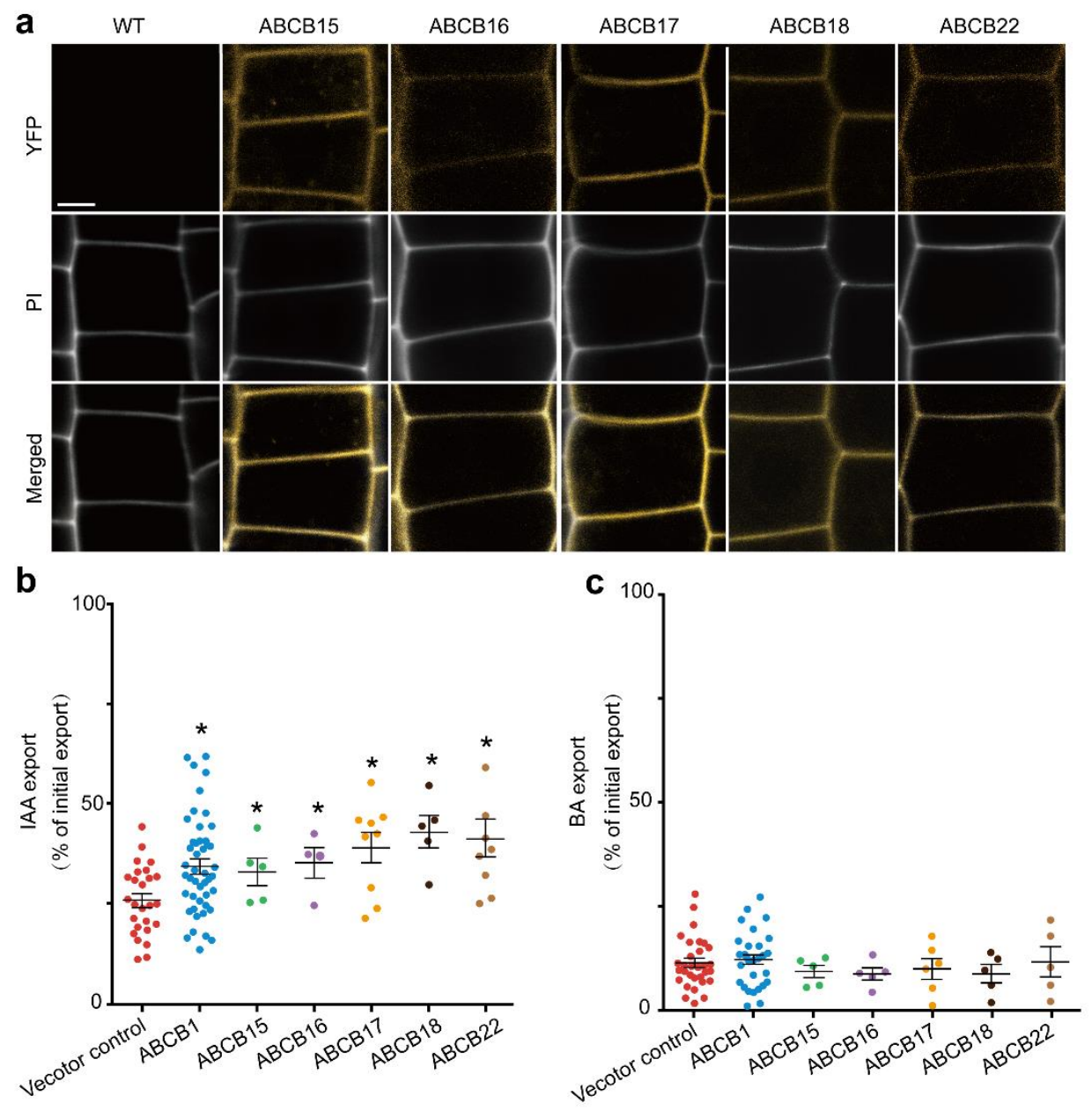
Epidermal YFP fluorescence in primary root meristems of 3-day-old WT, pro35S:YFP-ABCB15, pro35S:YFP-ABCB16, pro35S:YFP-ABCB17, pro35S:YFP-ABCB18, pro35S:YFP-ABCB22. Cell walls are stained by Propidium lodide $(\mathrm{PI})$ in grey. Scale bars represent $10 \mu \mathrm{m}$. All pictures were analyzed using the same magnification. b, $\mathbf{c} I A A$ and BA export assay. Export of radiolabeled IAA (b) and benzoic acid BA (c) assayed in parallel from tobacco mesophyll protoplasts expressing indicated $A B C B s$ of subgroup III against vector control. * indicates $\mathrm{P}<0.05$ (unpaired t-test with Welch's correction) (mean $\pm S E ; n \geq 4$ transport experiments generated from independent tobacco transfections). 
a

$$
\begin{aligned}
& 0 \\
& 0 \\
& 0 \\
& 0 \\
& 0 \\
& 01 \\
& 0 \\
& 0 \\
& 0 \\
& 2 \\
& 01 \\
& 0 \\
& 0 \\
& 0 \\
& 0 \\
& 0 \\
& 0 \\
& 0
\end{aligned}
$$
DAG1

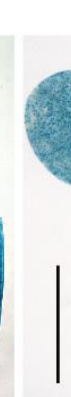

C

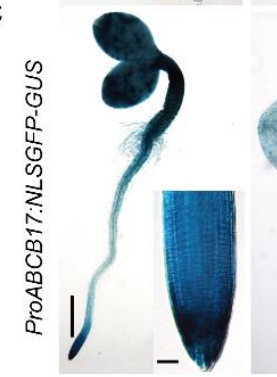

e
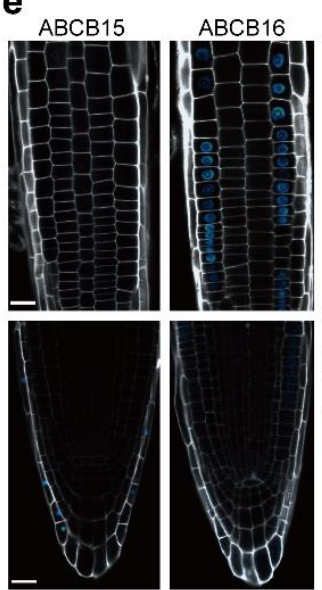

DAG3
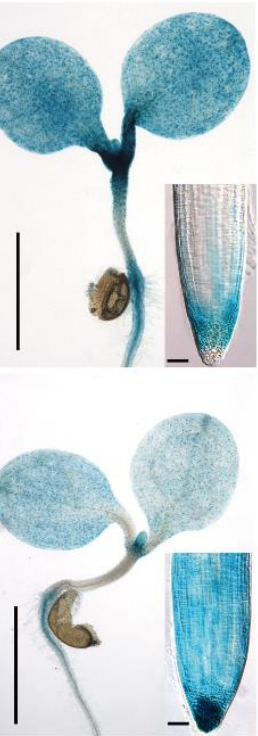
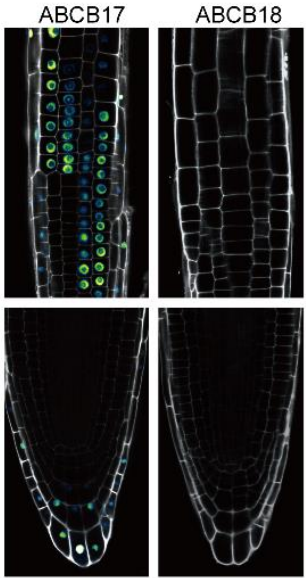

b

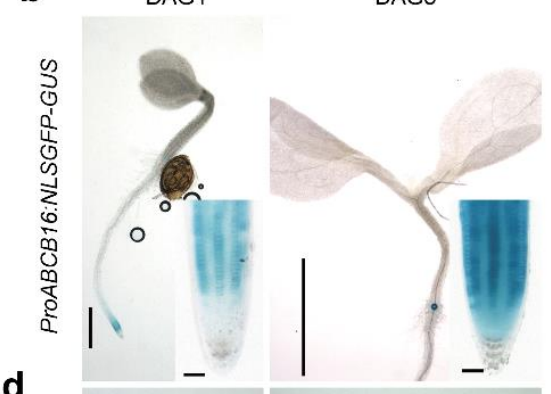

d

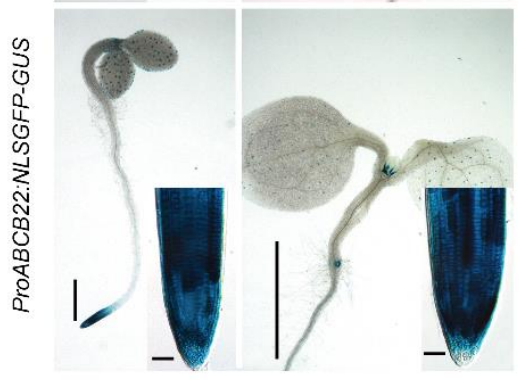

$A B C B 22$

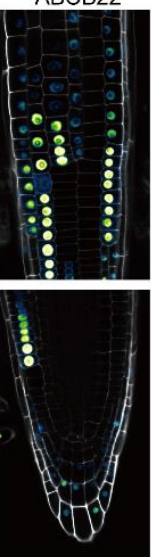

f

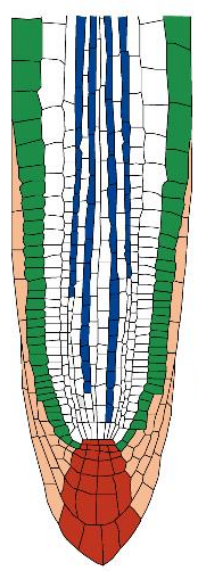

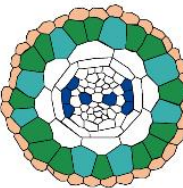

- $\mathrm{ABCB} 16$ $A B C B 16,17,22$ $A B C B 17,22$

A $\mathrm{ABCB} 15,17,22$

- $\mathrm{ABCB} 17,22$

Fig. 4: Overview of the expression patterns of subgroup III $A B C B s . a, b, c, d$ GUS expression pattern of proABCB $(15,16,17,18,22)$ :NLSGFP-GUS in 1- and 3-day-after germination seedlings (DAG). Scale bars = $0.5 \mathrm{~mm}$, for inset $=20 \mu \mathrm{m}$. e Surface and median view of GFP fluorescence in proABCB15:NLSGFPGUS, proABCB16:NLSGFP-GUS, proABCB17:NLSGFP-GUS, proABCB18:NLSGFP-GUS, and proABCB22:NLSGFP-GUS expression in roots of 3-day-old seedlings. Propidium iodide in grey. Scale bars represent $20 \mu \mathrm{m}$. f Summary of $A B C B$ subgroup III expression domains in the root apical meristem indicated on a longitudinal and radial section. 

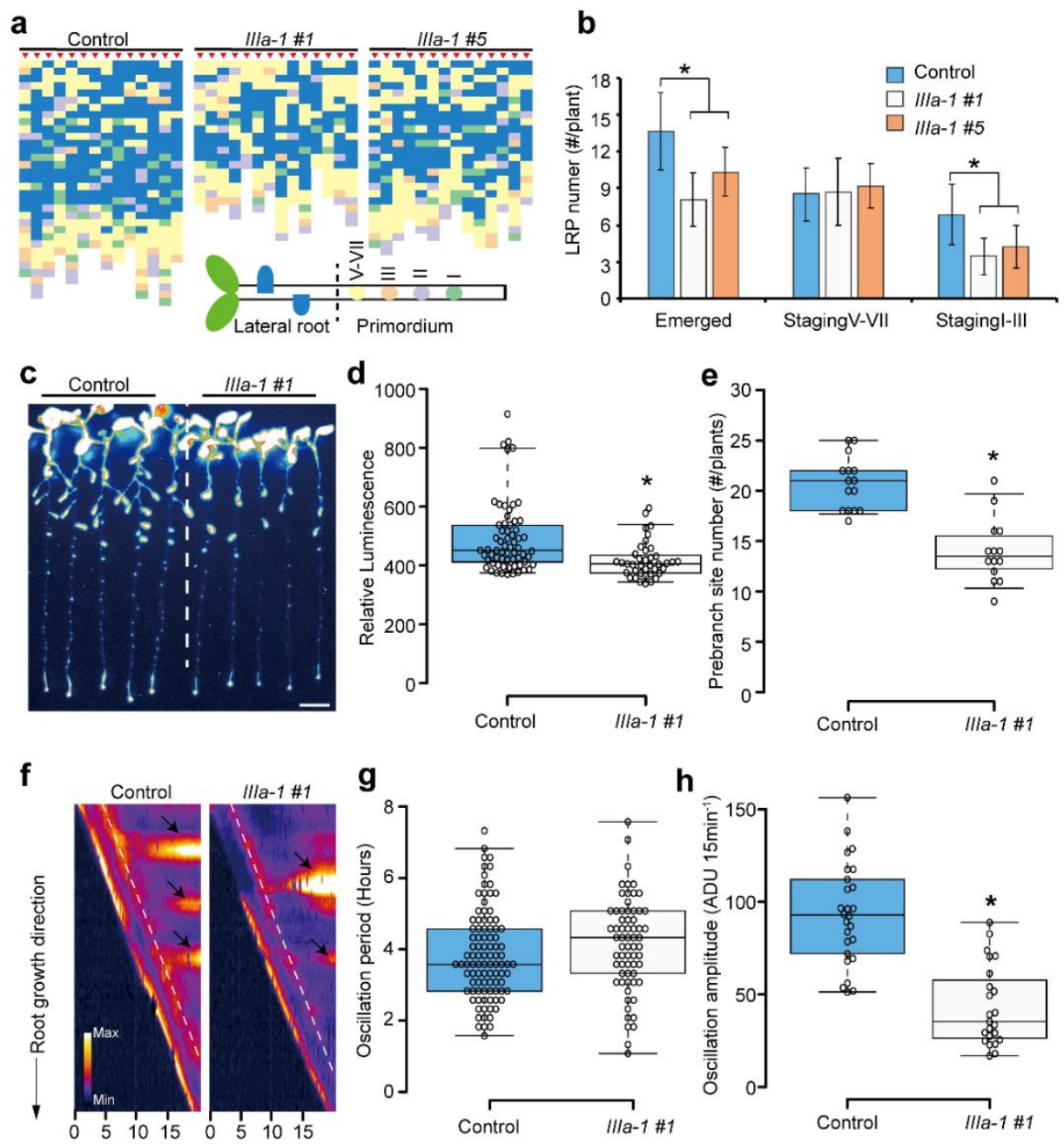

Figure 5: Subgroup III ABCBs control lateral root spacing by regulating pre-branch site number and the auxin oscillation amplitude. a Schematic representation of the distribution of emerged LRS and LRP along the primary root in 8-day-old WT and AtTAS1C-IIla (Line1 and line5) seedlings $(n=10)$. The color code corresponds to different LRP stages as indicated in the schematic root. Each column represents an individual root indicated by red arrowheads. b Quantification of different LR stages and emerged LRs in $(\mathbf{a})$, shown are averages $( \pm S D)(n=10)$. c Pre-branch sites in 8-day-old WT and AtTAS1c-Illa line1 as determined by DR5:LUC luminescence. Scale bar represent $0.8 \mathrm{~cm}$. d Quantification of DR5:LUC luminescence intensity of the pre-branch sites in 8-day-old WT $(n=64)$ and AtTAS1C-IIla line1 (IIla-1 \#1; $\mathrm{n}=39$ ) seedlings. e Quantification of pre-branch site number per root in (c). $\mathrm{n}=15$ (WT), 14 (IIla-1 line 1). f Kymograph of DR5:LUC intensity along the primary root in 3-dayold WT and AtTAS1c-Illa line1 seedlings over $20 \mathrm{hr}$. DR5:LUC luminescence intensity is color coded (see color code in the bottom left corner of the panels) and plotted following the primary root elongation (y-axis) and time (x-axis). The arrows highlight pre-branch sites and white dashed lines indicate the DR5:LUC signal in OZ. $\mathbf{g}, \mathbf{h}$ Quantification of the oscillation period (g) and amplitude (h) of DR5:LUC in 3-day-old WT and AtTAS1c-IIla (line1) $(n=24)$. Center lines in box plots show the medians; box limits indicate the 25th and 75th percentiles as determined by $R$ software; whiskers extend to 5 th and 95th percentiles, outliers are represented by dots; data points are plotted as open circles. * indicates $P<0.05$ (two-tailed Student's t-test) 
a
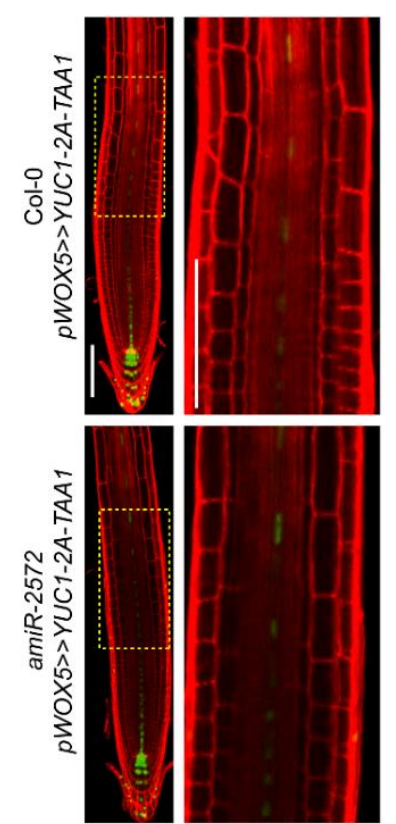

$\mathrm{Oh}$

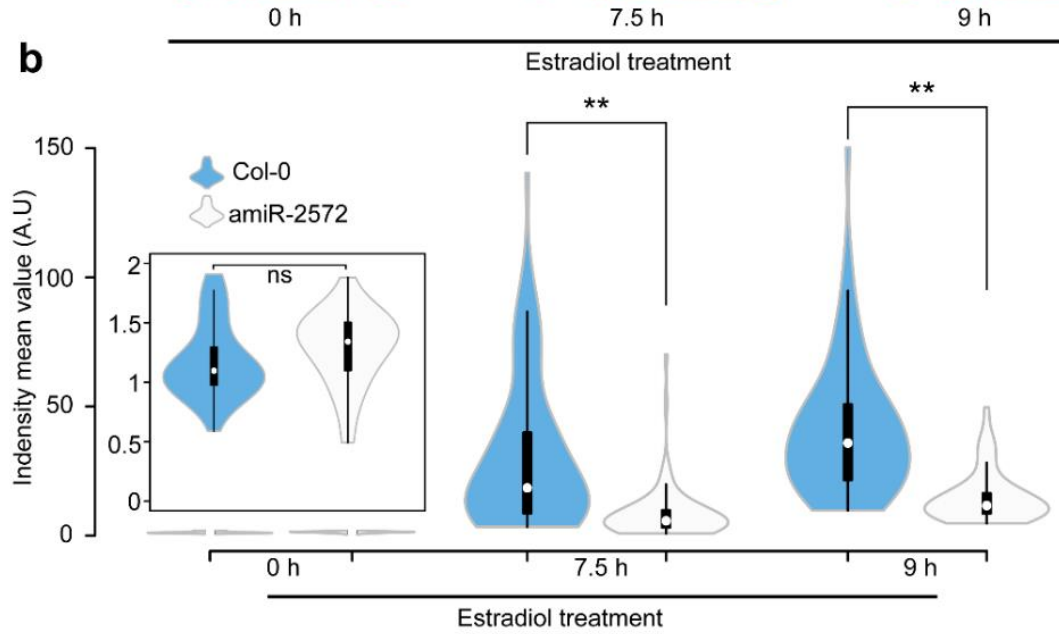

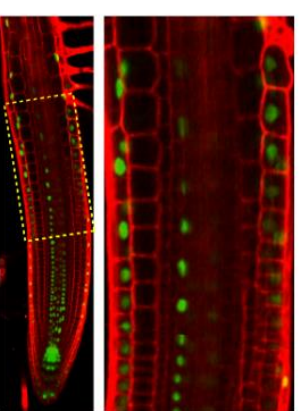
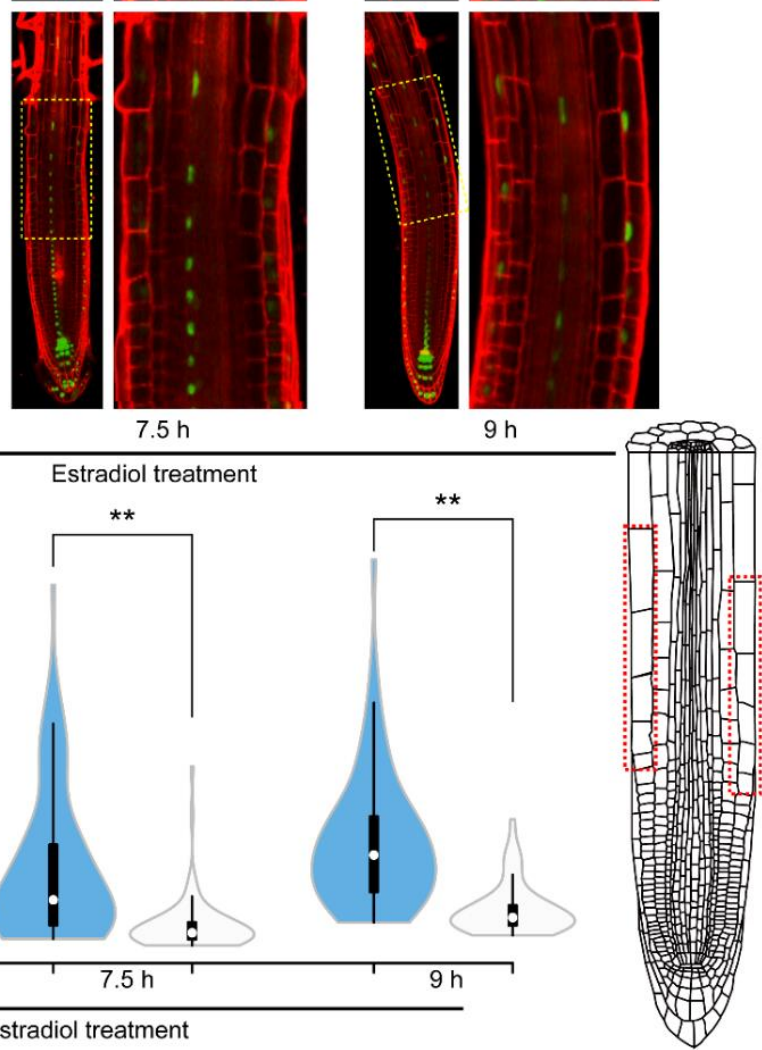

Figure 6 Subgroup III ABCBs contribute to shootward auxin transport in the LRC and meristem.

a Analysis of DR5:VENUS expression in the root elongation zone of 4-day-old $p$ WOX5 >>YUC1-2ATAA1, in Col-0 and amiR-2572 treated with $\beta$-estradiol $(5 \mu \mathrm{M})$ for $0,7.5$ and $9 \mathrm{~h}$. Propidium iodide in red. Yellow squares indicate zoomed pictures. Scale bar $=100 \mu \mathrm{M}$. b Quantification of DR5:VENUS signals in the epidermis of the elongation zone, as indicated in the scheme (Red square). White dots indicate the medians; box limits indicate the 25th and 75th percentiles as determined by R software; whiskers extend 1.5 times the interquartile range from the 25th and 75th percentiles; polygons represent density estimates of data and extend to extreme values. 10 cells in the elongation zone per seedling and at least 4 seedlings of each treatment were measured ** indicates $\mathrm{P}<0.01$ (two-tailed Student's t-test). 